\title{
Management of HIV-associated focal brain lesions in developing coun- tries - experience at Chris Hani Baragwanath Hospital
}

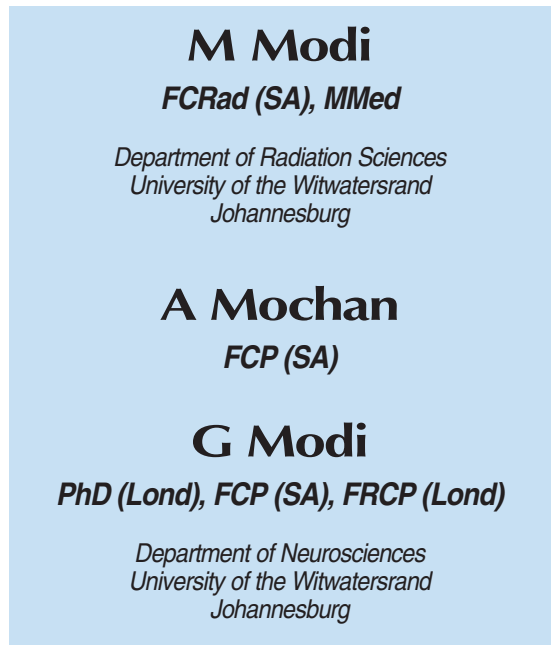

\section{Abstract}

Background. HIV-associated focal brain lesions (FBLs) are caused by opportunistic infections, neoplasms, or cerebrovascular diseases. In developed countries toxoplasma encephalitis (TE) is the most frequent cause followed by primary CNS lymphoma (PCNSL). Guidelines based on these causes have been proposed and successfully implemented. These guide- lines do not consider the causes of HIV-associated FBL in developing countries where treatable infections predominate. The guidelines as proposed would adversely influence outcome if applied to patients in developing countries.

Objective. To determine a practical approach to the management of HIVassociated FBLs in developing countries.

Design. Prospective case series.

Methods. Management based on presumed aetiologies of the FBLs determined by collating information obtained from computed tomography (CT) scans, cerebrospinal fluid and blood studies, concurrent nonneurological illness and response to treatment.

Results. The principal presumed cause of FBL was tuberculosis (TB) (69\%), and the therapeutic response was good in $69 \%$ of patients.

Conclusions. In developing countries infections are the predominant cause of HIV-associated FBL. The principal cause is determined by the infection that is endemic to the population being studied. Empiric treatment based on limited investigations should be directed according to the nature of this infection. A modified algorithm is proposed.

\section{Introduction}

Focal brain lesions (FBLs) caused by opportunistic infections, neoplasms, or cerebrovascular diseases are common neurological consequences of HIV infection.

In developed countries toxoplasma encephalitis (TE) is the most frequently identified cause of HIV-associated FBL, followed by primary CNS lymphoma (PCNSL).,2 On this basis the Quality Standards Subcommittee of the American Academy of Neurology (AAN) proposed that HIV-positive patients presenting with FBL should be empirically treated for toxoplasmosis initially. ${ }^{1}$ Failure to improve clinically or radiologically over the succeeding 10- to 14-day period warrants stereotactic brain biopsy in order to institute specific and appropriate therapy. ${ }^{1}$ An alternative non-invasive approach that is otherwise similar is based on the fact that brain biopsies do not influence survival in these patients. ${ }^{2}$ With this approach diagnosis is determined by the response to anti-toxoplasma treatment. ${ }^{2}$ Failed responders are reviewed and presumed to have PCNSL or toxoplasmosis resistant to standard therapy and treated appropriately. ${ }^{2}$ These guidelines and approaches to the management of HIV-associated FBL have been effective in developed countries.

In developing countries infections are the main cause of FBL associated 
with HIV. ${ }^{3-10}$ Studies from African and Central American countries describe either tuberculosis (TB) or toxoplasmosis as the two most frequently occurring causes.-8 These observations have been corroborated in similar studies from India. ${ }^{9} 10$ The relative frequencies with which these infections cause FBL are not well documented. The nature of the HIV-associated FBL is probably determined by the infection/s that are endemic to the population being studied. ${ }^{7} \mathrm{~TB}$ is endemic in developing countries. ${ }^{11}$ This therefore implies that if the AAN or British guidelines are strictly applied to developing regions where $\mathrm{TB}$ is endemic large numbers of patients will be inappropriately managed.

We therefore prospectively studied and managed 32 patients with HIVassociated FBL. Our approach was to presume the most likely cause in our setting determined by collating information obtained from computed tomography (CT) scans, cerebrospinal fluid (CSF) and blood studies, concurrent non-neurological illness and response to treatment. We did not emphasise the need for accurate histological diagnosis of the FBL.

\section{Study design}

This was a prospective case series.

\section{Patients and methods}

We studied 32 HIV-infected patients (older than 18 years) with FBL as diagnosed on CT scans. The study was carried out at the Chris Hani Baragwanath Hospital (CHBH) in Soweto, South Africa. Patients were recruited over a 12-month period.
$\mathrm{CHBH}$ is a 3 300-bed public university hospital that serves a predominantly black urban population of approximately 3 million people.

The patients were all inpatients, admitted to the medical wards of the $\mathrm{CHBH}$.

All patients in the study were black, heterosexual, non-intravenous drug abusers and were anti-retroviral therapy-naive. None of the patients was on prophylactic treatment for Pneumocystis carinii or toxoplasma.

The following data were recorded.

Demographics: Age, sex, ethnic group, medication/drug use, and associated non-neurological illnesses.

Blood: Full blood count, erythrocyte sedimentation rate (ESR), glucose, urea and electrolytes, serum calcium, phosphate and magnesium, liver function tests, T-cell subsets, HIV viral loads (HIV-1 RNA), serological tests for syphilis (WR, TPHA, FTAAbs), cysticercosis (ELISA), Toxoplasma gondii (CFT), typhoid (TMX), viral studies for hepatitis A, B and C, cytomegalovirus (CMV), HTLV-I and blood cultures.

\section{Cerebrospinal fluid (CSF):} Chemistry, cell counts, cytology, and adenosine deaminase (ADA) level. TB ELISA and polymerase chain reaction (PCR), syphilis, cysticercosis and toxoplasma serology, HIV viral load (HIV-1 RNA), PCR for herpes simplex, varicella zoster, cytomegalovirus (CMV), Epstein-Barr virus (EBV) and herpes simplex type 6, India ink staining, cryptococcal antigen, bacterial (including Mycobacterium tuberculosis) and fungal cultures.

Sputum: Cytology, microscopy, culture and sensitivity, and acid-fast bacilli (AFB).

Radiology: Chest X-rays (CXRs).
CT scans without and with contrast enhancement were done prior to treatment and repeated days or weeks later depending on the patients' clinical condition.

Presumed diagnosis of FBL: The accurate and definitive diagnosis of FBL is dependent on histopathology. In this study as we were unable to obtain histopathological diagnosis we presumed the diagnosis of the FBL by collating information obtained from the CT scans (at the time of presentation and on empiric treatment), CSF and blood studies, CXR findings, associated non-neurological illness/es and response to treatment.

Presumed diagnosis of TB was based on some or all of the following: (i) CT brain scan appearance, i.e. hypodense or isodense rounded lesions with irregular walls of varying thickness, oedema and mass effect, cortical location, ring or nodular enhancement, and increased basal meningeal enhancement; (ii) positive TB culture in the CSF; (iii) pulmonary TB (PTB) on CXR and/or $\mathrm{AFB}$ on sputum microscopy; (iv) increased protein, decreased glucose, pleocytosis (lymphocytes and/or polymorphonuclear cells), positive TB ELISA, PCR in the CSF; $(v)$ negative toxoplasma CFT; and (vi) response to $\mathrm{TB}$ treatment, clinically and radiologically.

Neurosyphilis was diagnosed on the basis of CSF serology (WR, TPHA, FTA).

Presumed diagnosis of toxoplasmosis was based on some or all of the following: (i) positive toxoplasma CFT; (ii) CD4+ count less than 100 cells $/ \mathrm{mm}^{3}$; (iii) hypodense, multiple (> 5) rim-enhancing lesions with oedema, with basal ganglia and 
grey/white matter zones as common sites; and (iv) response to toxoplasma treatment.

Diagnosis of neurocysticercosis was based on some or all of the following: (i) regular thin-walled, cystic, ring-enhancing lesions (often with a scolex); (ii) intracranial calcifications; and (iii) positive blood and/or CSF serology (ELISA).

Cryptococcosis was diagnosed on CSF studies (India ink, latex agglutination, fungal cultures).

Presumed diagnosis of PCNSL was based on some or all of the following: (i) hyperdense lesion/s, often confluent, with no oedema, homogeneous contrast enhancement, periventricular/subependymal in location, corpus callosum involvement; (ii) CD4+ count less than 100 cells $/ \mathrm{mm}^{3}$; (iii) atypical lymphocytes in the CSF; (iv) EBV PCR-positive in CSF; and $(v)$ no response to anti-TB or anti-toxoplasma treatment.

Presumed diagnosis of progressive multifocal leucoencephalopathy (PML) was based on some or all of the following: (i) lesions confined to white matter, hypodense, nonenhancing appearance on CT scan, T2 hyperintense and $\mathrm{T} 1$ hypointense signal on MRI scans if performed, no mass effect; and (ii) JC (John Cunningham) virus PCR-positive in CSF.

Treatment: Patients were treated with specific medication based on the presumed diagnosis: TB (isoniazid, rifampicin, pyrazinamide, ethambutol), neuroyphilis (IVI penicillin G), toxoplasmosis (sulfadiazine, pyrimethamine), and cysticercosis (albendazole).

If response was good, the initial treatment regimen was continued. If response was poor, a new or second presumed diagnosis was entertained, and additional treatment for this diagnosis was instituted. The additional or new treatment was as described above.

A good response was one in which the patient showed ongoing improvement measured radiologically by a reduction in size and/or number of lesions on scan.

A poor response was one in which the patient showed no improvement with no change or worsening of the FBL on scan.

\section{Results (Tables I - III)}

\section{Age and sex}

The age range was 18 - 49 years (mean 33.5 years), with 19 male patients and 13 female patients (male/female 1.5:1).

\section{Clinical presentation}

On CNS examination there were focal signs in 21 patients (66\%), normal in 9 patients (28\%), dementia in 6 patients (19\%), and encephalopathy in 2 patients $(6 \%)$.

Patients were examined clinically on admission by the authors GM or AM who were blinded to the CT scan result. Once patients were recruited into the study the follow-up examinations done by either GM or AM were not blinded.

\section{Non-neurological illnesses}

Non-neurological illnessess were present in 27 patients (84\%): active PTB (19 patients $(60 \%)$ ), active PTB and tuberculous breast abscess (1 patient), tuberculous lymphadenitis (1 patient (diagnosed on fine needle aspirate)), Pneumocystis carinii pneumonia (PCP) (1 patient (diagnosed on sputum cytology)), and pos- itive syphilis blood serology (WR, TPHA) (11 patients).

\section{CD4 counts and staging}

Staging was determined using the Centers for Disease Control (CDC) 1993 revised classification system for HIV infection and AIDS-defining illnesses. $^{12}$

The patients had CD4+ T-lymphocyte counts ranging from 1 to 768 cells $/ \mathrm{mm}^{3}$. Four patients $(12 \%)$ had CD4+ counts of greater than 500 cells $/ \mathrm{mm}^{3}$. Four patients $(12 \%)$ had CD4+ counts between 200 and 500 cells $/ \mathrm{mm}^{3}$. Twenty-four patients (76\%) had CD4+ counts of less than 200 cells $/ \mathrm{mm}^{3}$.

\section{HIV viral loads}

HIV blood viral loads were obtained in 29 of the 32 patients. These ranged from 10750 copies $/ \mathrm{ml}$ to $>750000$ copies $/ \mathrm{ml}$.

\section{CSF results}

There was elevated CSF protein, with or without pleocytosis in 23 patients. Cysticercosis ELISA was positive in 8 patients, TB ELISA was positive in 1 patient, TB PCR was positive in 1 patient, $\mathrm{TB}$ culture was positive in 1 patient, cryptococcal antigen was positive in 1 patient, syphilis serology was positive in 1 patient, atypical Bcells were present in 1 patient, and toxoplasma immunoglobulin $\mathrm{G}(\operatorname{IgG})$ was positive in 1 patient, normal in 7 patients $(22 \%)$, and not performed in 2 patients for practical reasons.

\section{Radiology}

\section{Chest X-rays}

Features of PTB were present in 20 patients $(63 \%)$, there were bilateral peri-hilar reticulo-nodular infiltrates in 1 patient, and CXR was normal in 11 patients (34\%). 


\section{CT scans}

These were performed on admission and repeated days or weeks later depending on the patient's clinical condition. The following observations were made: 12 patients had solitary lesions (38\%) and 20 patients had multiple lesions $(62 \%)$.

In the 12 patients with solitary lesions the CT scans were suggestive of TB in 8 patients, neurocysticercosis in 3 patients and PCNSL in 1 patient. In the 20 patients with multiple lesions the CT scans were suggestive of $\mathrm{TB} /$ toxoplasmosis (12 patients), neurocysticercosis (3 patients), PML (1 patient), and $\mathrm{TB} /$ toxoplasmosis and neurocysticercosis (2 patients). Two patients had cerebral infarcts.

Meningeal enhancement (Fig. 1) was present in 5 of the 12 patients with solitary lesions and in 12 of the 20 patients with multiple lesions. None of the patients diagnosed radiologically as neurocysticercosis, PCNSL or PML had meningeal enhancement.

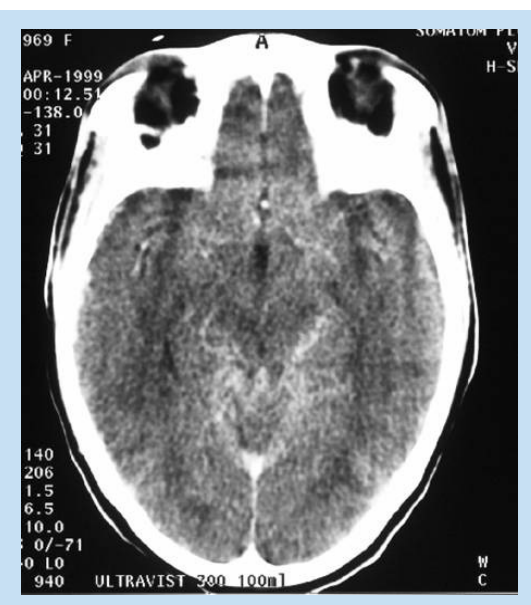

Fig.1. CT scan showing basal meningeal enhancement in a patient with presumed TB.

The radiological diagnoses were therefore TB (17 patients), neurocysticercosis (6 patients), PML (1 patient), PCNSL (1 patient), $\mathrm{TB} /$ toxoplasmosis and neurocysticer- cosis (2 patients), TB/toxoplasmosis ( 3 patients), and in 2 patients cerebral infarcts (both had basal meningeal enhancement).

\section{Presumed causes}

Presumed diagnoses were determined after correlating the radiological diagnosis with blood, CSF and non-neurological manifestations.

The 2 patients with cerebral infarctions had meningitis. In 1 patient TB meningitis (TBM) was confirmed by culture positivity, and in the other cryptococcosis was identified on India ink stains and confirmed by fungal cultures and latex antigen tests.

Of the 17 patients with radiological diagnosis of presumed TB, 14 patients had active PTB (sputum AFB-positive), 1 patient had histologically confirmed TB adenitis, 1 patient had PCP and 1 patient had no nonneurological disease. The CSF and blood studies in these patients showed no specific diagnostic abnormalities other than neurosyphilis in 1 patient. $\mathrm{TB}$ cultures were negative in all 17 patients. One patient had a positive TB PCR in the CSF. This patient had PTB and a solitary enhancing FBL. As indicated above, all 17 patients had increased basal meningeal enhancement. In this group correlating the radiological diagnosis with blood, CSF and non-neurological manifestations did not alter the diagnosis. These patients were therefore treated with the 4-drug anti-TB regimen as firstline treatment.

Five patients had radiological diagnoses of TB/toxoplasmosis (2 of whom had associated neurocysticercosis). In these 5 patients, 1 had positive toxoplasma serology in the blood and CSF. This patient had multiple enhancing FBL (including lesions suggestive of neurocysticercosis) on CT scan with basal meningeal enhancement. The patient also had PTB. One patient had a positive blood toxoplasma serology, no PTB, and multiple enhancing FBL with no basal meningeal enhancement on CT scan. The 3 other patients had PTB, negative toxoplasma serology, multiple enhancing FBL with no basal meningeal enhancement (including lesions suggestive of neurocysticercosis in 1 patient) on CT scan. In this group correlating the radiological diagnosis with blood, CSF and nonneurological manifestations influenced the presumed diagnosis. One of these 5 patients was therefore diagnosed with isolated toxoplasmosis and treated with anti-toxoplasmosis treatment as first-line therapy. Of the remaining patients in this group, 2 were classed as TB, 1 as TB and neurocysticercosis, and 1 as TB, toxoplasmosis and neurocysticercosis, and treated accordingly.

The following presumed diagnoses were therefore made: 17 patients (53\%) TB, 6 patients (19\%) neurocysticercosis, 2 patients (6\%) TB and neurocysticercosis, 2 patients $(6 \%)$ multiple infarcts (1 TBM, 1 cryptococcal meningitis), 1 patient TB and neurosyphilis, 1 patient toxoplasmosis, 1 patient toxoplasmosis, TB and neurocysticercosis, 1 patient PML, and 1 patient PCNSL.

In the patients with multiple infections as diagnoses, these were based on positive serology in the case of neurosyphilis, positive serology and CT scan features in the case of neurocysticercosis, basal enhancement on CT scan with non-neurological TB in the case of $\mathrm{TB}$, and positive serology in the case of toxoplasmosis. 
Treatment was instituted for each infection at the outset.

\section{Response to empirical treat- ment initiated}

The following overall response to treatment was obtained (Table III): (i) good response 22 patients (69\%); (ii) poor response 3 patients (9\%); and (iii) demised 7 patients (22\%).

In the patients with a presumed diagnosis of TB and treated only with anti-TB treatment (17 patients): 12 patients had a good response (Fig. 2) (CD4+ counts 21 to 610 cells $\left./ \mathrm{mm}^{3}\right), 3$ patients had a poor response (CD4+ counts 1,55 , and 180 cells $\left./ \mathrm{mm}^{3}\right)$, and 2 patients demised (CD4+ counts 6,14 cells $/ \mathrm{mm}^{3}$ ).

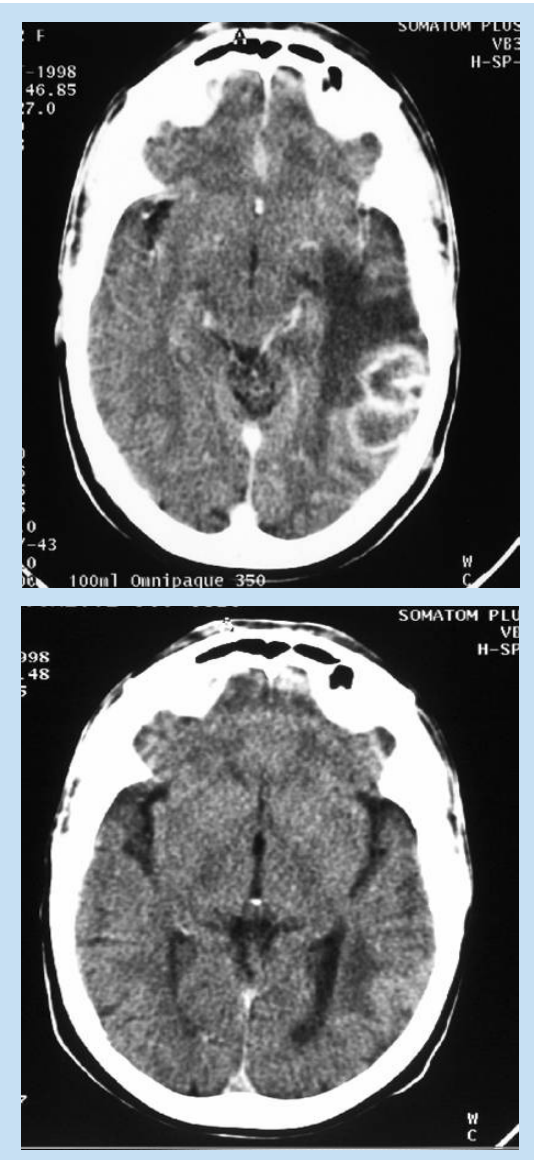

Fig. 2a and $b$. CT scans of a patient with presumed TB at presentation (top) and on antiTB treatment after 6 weeks (bottom).
The 6 patients with neurocysticercosis (CD4+ counts 106 - 768 cells $/ \mathrm{mm}^{3}$ ) responded well to treatment.

The 2 patients with infarctions as FBL (CD4+ counts of 96 and 240 cells $/ \mathrm{mm}^{3}$ ) demised.

The patient treated as isolated toxoplasmosis $\left(\mathrm{CD} 4+\right.$ count 8 cells $\left./ \mathrm{mm}^{3}\right)$ demised.

In the mixed infection group (2 TB and neurocysticercosis, 1 TB and neurosyphilis, $1 \mathrm{~TB}$, toxoplasmosis and neurocysticercosis) response to treatment was good in all 4 cases (CD4+ counts of $30-104$ cells $/ \mathrm{mm}^{3}$ ).

The patients diagnosed with PCNSL (CD4+ count 22 cells $/ \mathrm{mm}^{3}$ ) and PML (CD4+ count 7 cells $\left./ \mathrm{mm}^{3}\right)$ demised. The patient with presumed PML had autopsy confirmation of this diagnosis.

The 3 poor responders to first-line TB treatment (all presumed TB) received additional anti-toxoplasmosis treatment and broad-spectrum antibiotics. There was no clinical or radiological change in spite of these added treatments during the followup period.

\section{Discussion}

The FBLs seen in our patients were determined to be almost exclusively infectious in nature. Of the 32 patients only 1 patient was presumed to have a non-infectious aetiology (PCNSL). Four patients (12\%) were diagnosed with dual/multiple infections. Dual/multiple CNS infections are not common in HIV but have been described. ${ }^{13}$

We determined the principal presumed cause of FBL in our patients to be TB.

The key features differentiating suspected TB from other infectious aetiologies were: concurrent presence of PTB or other non-neurological TB, and basal meningeal enhancement.

The FBLs in the patients with presumed TB were predominantly cortical, with thick irregular walls, hypodense centres, and rim enhancement. Despite detailed analysis, of all the parameters used the only possible differentiating radiological feature was the presence of basal meningeal enhancement in TB (Table II). Of the 22 patients presumed to have $\mathrm{TB}$ (alone or in combination with other infections), basal meningeal enhancement was present in 16 patients (72\%). The patients with presumed toxoplasmosis and neurocysticercosis had no basal meningeal enhancement. Patients with multiple infections who had basal meningeal enhancement all had TB as one of the presumed infections. The only patient with basal meningeal enhancement whose presumed diagnosis was not $\mathrm{TB}$, had cryptococcal meningitis with cortical infarctions as the FBL. TB cultures were not helpful in the acute management of our patients. Only 1 of our patients had a positive CSF TB culture. This was the patient with TBM and cortical infarctions as the FBL. CSF TB PCR and ELISA were also of no value in our study, being positive in only 1 patient respectively.

In terms of non-neurological TB, this was diagnosed in 21 (66\%) of our patients, of whom 19 had active PTB. TB is endemic in our population. ${ }^{8,11} \mathrm{In}$ the Gauteng province of South Africa in 1999, the incidence of TB was $270 / 100000$ population. In 2001 the incidence was $315 / 100$ 000. РTB accounts for $75 \%$ and extra-pulmonary TB accounts for $25 \%$ of cases (Gauteng Department of Health - 
unpublished data obtained from $\mathrm{Dr}$ Riana Louw, with permission). Sixtythree per cent of our patients had PTB. It is therefore not surprising that our presumed diagnoses showed a bias towards TB.

With regard to toxoplasmosis we found that only 2 of our patients had positive serology ( 1 in serum, 1 in CSF and serum). Prevalence figures for toxoplasmosis in our region and South Africa as a whole have been published. In Gauteng the prevalence amongst blacks is $29 \%,{ }^{14}$ and in
KwaZulu-Natal the prevalence is $46 \%$ in black pregnant women, the highest in the country. ${ }^{15}$ It may be, as is widely recognised, that serological tests are falsely negative in patients with advanced immunosuppression. However, active non-neurological toxoplasmosis has not been described in our population. We were unable to find data for toxoplasma retinitis, toxoplasma septicaemia, and toxoplasma pneumonitis in our adult population (with or without HIV). None of our patients had evidence of toxoplasmo- sis outside the nervous system. The KwaZulu-Natal study found toxoplasmosis as the main cause of HIV associated FBL. ${ }^{3}$ In another South African series of 38 HIV-infected patients with meningitis, toxoplasma IgG was positive in 14 patients (37\%). ${ }^{16}$ Two of these 14 patients also had FBL, both of which were TB (one confirmed histologically) and responded to TB treatment. ${ }^{16}$ The only other study from this country on HIV-associated FBL concurred with our findings that TB is the commonest cause of HIV-associated

\begin{tabular}{|c|c|c|c|c|c|}
\hline Patient data & $\begin{array}{l}\text { Neurological } \\
\text { examination }\end{array}$ & $\begin{array}{l}\text { Non-neurological } \\
\text { illnesses }\end{array}$ & CSF results & $\begin{array}{l}\text { CD4+ count } \\
\left(\text { cells } / \mathrm{mm}^{3}\right)\end{array}$ & Presumed cause \\
\hline $\mathrm{N}=32$ & Focal signs $66 \%$ & PTB 60\% & Elevated protein $59 \%$ & $72 \%:<200$ & $53 \% \mathrm{~TB}$ \\
\hline $\mathrm{M} / \mathrm{F}$ ratio $1.5: 1$ & Normal 28\% & $\begin{array}{l}\text { PTB }+ \text { TB breast } \\
\text { (1 patient) }\end{array}$ & Pleocytosis 9\% & 13\%: $200-500$ & $19 \%$ NCC \\
\hline \multirow[t]{6}{*}{ Mean age 33.5 yrs } & Dementia 19\% & $\begin{array}{l}\text { TB lymphadenitis } \\
\text { (1 patient) }\end{array}$ & TB PCR/Elisa + 6\% & $15 \%:>500$ & $3 \%$ Toxoplasmosis \\
\hline & Encephalopathy $6 \%$ & PCP (1patient) & \multicolumn{2}{|l|}{ Cryptococcal Ag $+3 \%$} & $3 \% \mathrm{PML}$ \\
\hline & & \multicolumn{3}{|c|}{ Syphilis (11 patients) Cysticercosis Elisa $+21 \%$} & $3 \%$ PCNSL \\
\hline & & \multicolumn{3}{|c|}{ Syphilis $+3 \%$} & $6 \%$ Infarcts \\
\hline & & & \multicolumn{2}{|l|}{ Toxoplasma $+3 \%$} & 13\% Mixed \\
\hline & & & \multicolumn{3}{|l|}{ Normal 22\% } \\
\hline \multicolumn{6}{|c|}{$\begin{array}{l}\mathrm{CSF}=\text { cerebrospinal fluid, } \mathrm{TB}=\text { tuberculosis, } \mathrm{PTB}=\text { pulmonary tuberculosis, } \mathrm{PCP}=\text { Pneumocystis carinii pneumonia, } \mathrm{NCC}=\text { neurocysticercosis, } \mathrm{PCNSL}= \\
\text { primary central nervous system lymphoma, } \mathrm{PML}=\text { progressive multifocal leucoencephalopathy, } \mathrm{PCR}=\text { polymerase chain reaction, ELISA }=\text { enzyme linked } \\
\text { immunosorbent assay. }\end{array}$} \\
\hline
\end{tabular}

Table II. Imaging characteristics of focal brain lesions (FBLs)

\begin{tabular}{|c|c|c|c|c|c|c|}
\hline$N$ & Type of lesions & Site of lesions & Wall definition & Density relative to brain & $\begin{array}{l}\text { FBL enhancement } \\
\text { characteristics }\end{array}$ & $\begin{array}{l}\text { Basal meningeal } \\
\text { enhancement }\end{array}$ \\
\hline \multirow[t]{6}{*}{90} & Solitary $38 \%$ & Cortical 79\% & Regular & Hyperdense $6 \%$ & Ring 63\% & Present 53\% \\
\hline & Multiple $62 \%$ & Subcortical 11\% & Thick 0\% & Isodense $9 \%$ & Nodular 6\% & Absent $47 \%$ \\
\hline & & Basal ganglia 7\% & Thin 100\% & Hypodense $85 \%$ & Lobular 3\% & \\
\hline & & BS/cerebellar 3\% & Irregular & & Gyriform 5\% & \\
\hline & & & Thick 61\% & & Mixed 21\% & \\
\hline & & & Thin 39\% & & Nil 2\% & \\
\hline
\end{tabular}




\section{ORIGINAL ARTICLE}

\begin{tabular}{|c|c|c|c|c|c|}
\hline \multicolumn{6}{|c|}{ Table III. Treatment outcomes of focal brain lesions (FBLs) } \\
\hline Patients $(N(\%))$ & Response & $\begin{array}{l}\mathrm{CD} 4+\text { count } \\
\left(\mathrm{mean}-\text { cells } / \mathrm{mm}^{3}\right)\end{array}$ & Presumed cause & $\begin{array}{l}\mathrm{CD} 4+\text { count } \\
\left(\text { range - cells } / \mathrm{mm}^{3}\right)\end{array}$ & $\begin{array}{l}\text { HIV viral loads } \\
\text { (range - copies/ml) }\end{array}$ \\
\hline \multirow[t]{3}{*}{$22(69 \%)$} & \multirow[t]{3}{*}{ Good } & \multirow[t]{3}{*}{188} & ТВ 55\% & $21-610$ & $76000->750000$ \\
\hline & & & NCC $27 \%$ & $107-768$ & $10751-305123$ \\
\hline & & & $\mathrm{TB}+$ other $18 \%$ & $63-238$ & $288281->750000$ \\
\hline $3(9 \%)$ & Poor & 80 & TB $(100 \%)$ & $1-180$ & $647696->750000$ \\
\hline \multirow[t]{5}{*}{$7(22 \%)$} & \multirow[t]{5}{*}{ Demised } & \multirow[t]{5}{*}{28} & ТВ $44 \%$ & $6-40$ & $242000->750000$ \\
\hline & & & Toxoplasmosis $14 \%$ & 8 & ND \\
\hline & & & PCNSL $14 \%$ & 22 & $>750000$ \\
\hline & & & PML 14\% & 7 & $>750000$ \\
\hline & & & Cryptococcosis $14 \%$ & 96 & 228668 \\
\hline
\end{tabular}

FBL. ${ }^{4}$ Further studies are required to clarify this issue. In our patients the presence of positive toxoplasma serology was used as an indication for toxoplasmosis treatment.

Neurocysticercosis, alone or in combination with other infections occurred in 9 patients (28\%). This may be a chance association of endemic infections. ${ }^{17}$ In a case report of 4 patients from Zimbabwe, the occurrence of neurocysticercosis in HIV-positive patients was likewise suggested to be due to chance association in endemic regions or due to the effect of HIV on the host immune response to cysticercosis. ${ }^{17}$ Non-specific host factors (innate resistance) together with acquired immunity are known to have an effect on the outcome of the primary infection in experimental models of cysticercosis. ${ }^{17}$ Host immunity is also an important factor in limiting the occurrence of cysticercosis in humans. ${ }^{17}$ The immunodeficient state accompanying HIV infection might therefore increase the frequency and severity of neurocysticercosis. ${ }^{17}$ In our series the mean $\mathrm{CD} 4+$ count in patients with neurocysticercosis alone was 509 cells $/ \mathrm{mm}^{3}$. These patients were therefore not immunocompromised. This implies a chance association. Further studies in respect of this association will be needed.

In contradistinction to the histopathologically based American guidelines, ${ }^{1}$ the initial treatment in the majority of our patients, where diagnosis (presumed) was based on collating clinical information (as described above), was directed against TB. Only 1 patient had initial toxoplasmosis treatment alone. Patients with multiple infections were treated for these different infections. Sixty-nine per cent of patients improved clinically and radiologically. These patients had a mean CD4+ count of 188 cells $/ \mathrm{mm}^{3}$. Fifty-five per cent of these good responders were diagnosed with TB. Twenty-seven per cent of the good responders had neurocysticercosis. The remainder had dual/multiple infections associated with TB. There were 3 poor responders (9\%). The mean $\mathrm{CD} 4+$ count in these patients was 80 cells $/ \mathrm{mm}^{3}$. All 3 were diagnosed with TB. Seven of the 32 patients demised. These patients had a mean CD4+ count of 28 cells $/ \mathrm{mm}^{3}$. In these patients the diagnoses were $\mathrm{TB}$, toxoplasmosis, PML, PCNSL, and cryptococcosis.

The good responders had higher CD4+ counts than those who responded poorly or demised. There was no similar correlation with blood HIV viral loads. This may imply that our diagnoses were more accurate in patients with $\mathrm{CD} 4+$ counts in excess of 100 cells $/ \mathrm{mm}^{3}$ but that they were less accurate when the $\mathrm{CD} 4+$ counts were less than 100 cells $/ \mathrm{mm}^{3}$. The patients who demised or responded poorly did not improve clinically or radiologically once additional treatments were instituted as described above. The patients who demised deteriorated rapidly, with death occurring within a few days of presentation to hospital. This may therefore also imply that the poor responders 


\section{ORIGINAL ARTICLE}

and those who demised did so from advanced immune deficiency rather than incorrect diagnosis and treatment of the FBL.

A limitation of our study is the small number of patients and the lack of long-term follow-up. The patients who improved were discharged and continued treatment as outpatients. After a few consultations most were lost to further follow-up.

\section{Conclusions}

Our data demonstrate that applying the 'developed nations guidelines for HIV associated FBL' to a developing region could have a deleterious effect on outcome. This is largely because non-infectious aetiologies, namely PCNSL are uncommon and TE is not necessarily the most frequent infectious aetiology. We agree with the British proposal that brain biopsies are not necessary for treat- ment. The AAN guidelines are not possible to implement in developing regions, because of financial constraints and the limited availability of neurosurgical and neuropathological resources and services.

In developing countries HIV-positive patients who present with FBL should be treated initially with medication specific to the infection that is endemic to that population. In areas where TB is endemic our results indicate that anti-TB treatment should be

\section{HIV - FBL}

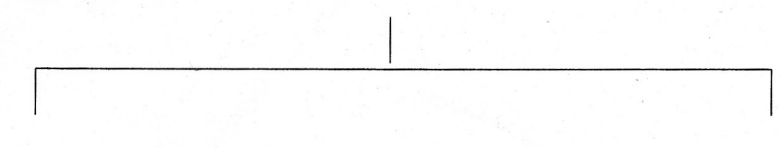

CYSTIC

SOLID

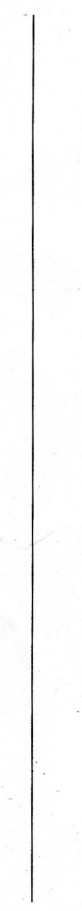

NCC
MENINGEAL ENHANCEMENT

ENHANCING

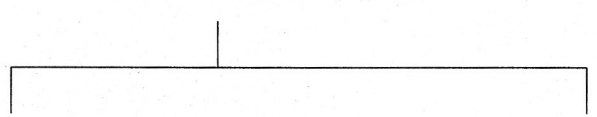

NO MENINGEAL ENHANCEMENT

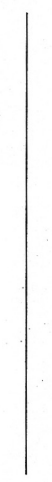

TB

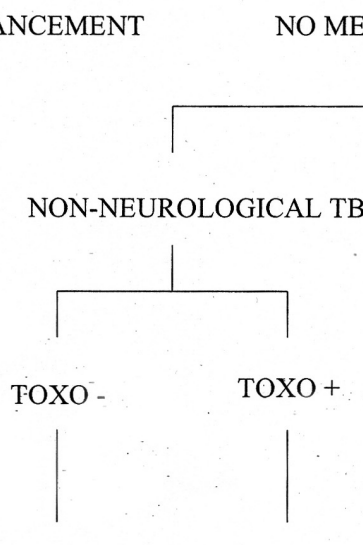

TB

TB/TOXO
NO NON-NEUROLOGICAL TB

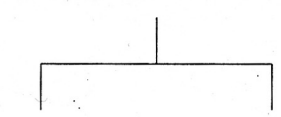

TOXO +

TOXO -

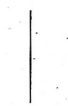

TOXO
NON-ENHANCING

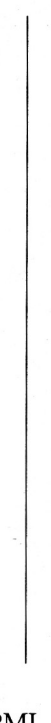

Fig.3. Algorithm for the initial assessment of HIV-associated focal brain lesions (FBLs) in developing regions. (TB =tuberculosis, NCC = neurocysticercosis, TOXO = toxoplasmosis, PCNSL = primary central nervous system lymphoma, PML = progressive multifocal leucoencephalopathy, $+=$ positive, = negative.) 
the initial treatment of known or suspected HIV-associated FBL. Further studies of this nature from other developing regions are needed to validate our approach especially with respect to the role of the endemic infection, which is treatable and would influence outcome and survival.

Perhaps the most important contribution of our study is that we show that detailed laboratory investigations (such as HIV viral loads, viral cultures, TB cultures, TB ELISA, TB PCR, and even CD4 counts) are not necessary to arrive at a reasonable presumed diagnosis of a FBL in HIVpositive patients in order to institute effective treatment. CT scans of the FBL, with the exception of neurocysticercosis and possibly PML, were found to be nonspecific. The most useful parameters that assisted us were non-neurological illness, toxoplasma serology, and meningeal enhancement on CT scans.

\section{Algorithm (Fig. 3)}

On the basis of our results we propose the following approach to the presumed diagnosis and thereby initial treatment of HIV-associated FBL in developing regions. The important parameters as discussed above are meningeal enhancement on CT scan, presence of non-neurological TB, and toxoplasma serology.

The FBLs are first categorised into solid or cystic. The cystic FBLs are indicative of neurocysticercosis (except in rare cases, e.g. hydatid). The solid FBLs are subdivided into enhancing or non-enhancing lesions. Lack of enhancement suggests PML. Enhancing FBLs are divided into those with and those without increased basal meningeal enhancement. The presence of increased basal meningeal enhancement is suggestive of TB. FBLs without increased meningeal enhancement are separated on the basis of accompanying nonneurological $\mathrm{TB}$ and toxoplasma serology (see algorithm). Initial treatment is instituted accordingly. Patients are monitored clinically. Good responders are maintained on this treatment. Poor responders are reassessed with CT scans. Presumed diagnosis and treatment is appropriately adjusted, e.g. addition of antitoxoplasma treatment, antibacterial treatment (pyogenic abscess).

A limitation of this approach is the requirement of a CT scan for evaluation. In developing regions where this is not available, it might be best advised to treat suspected FBL in HIV patients for TB (in endemic areas) and toxoplasmosis.

\section{References}

1. Quality Standards Subcommittee of the American Academy of Neurology. Evaluation and management of intracranial mass lesions in AIDS. Neurol 1998; 50: 21 - 26.

2. Sadler M, Brink NS, Gazzard BG. Management of intracerebral lesions in patients with HIV: a retrospective study with discussion of diagnostic problems. QJM 1998; 91: 205 - 217.

3. Bhigjee AJ, Naidoo K, Patel VB, Govender D. Intracranial mass lesions in HIV-positive patients - The KwaZulu/Natal experience. S Afr Med J 1999; 89: 1284-1288.

4. Smego RA, Orlovic D, Wadula J, Modi G. A diagnostic and therapeutic algorithm for CNS mass lesions in HIV/AIDS. S Afr J Epidemiol Infect 2000; 15: 7-13.

5. Trujillo JR, Garcia-Ramos G, Novak IS, Rivera VM, Huerta E, Essex M. Neurologic manifestations of AIDS: a comparative study of two populations from Mexico and the United States. $J$ Acquir Immune Defic Syndr Hum Retrovirol 1995; 8: 23-29.

6. Lucas SB, Hounnou A, Peacock C, et al. The mortality and pathology of HIV in a west African city. AIDS 1993; 7: 1569-1579.

7. Modi M, Modi G. New onset seizures in HIV infected patients - a review and guide to management. S Afr Med J 2001; 91: 1025-1026.

8. Karstaedt AS, Valtchanova S, Barriere R, CreweBrown HH. Tuberculous meningitis in South African urban adults. QJM 1998; 91: 743-747.

9. Santosh V, Shankar SK, Das S, et al. Pathological lesions in HIV positive patients. Indian J Med Res 1995; 101: 134-141.

10. Mathew MJ, Chandy MJ. Central nervous system toxoplasmosis in acquired immunodeficiency syndrome: An emerging disease in India. Neurol India 1999; 47: 182-187.

11. Raviglione MC. The TB epidemic from 1992 to 2002. Tuberculosis 2003; 83: 4-14.

12. Centers for Disease Control. 1993 revised classifications system for HIV infection and expanded surveillance case definition for AIDS among adolescents and adults. MMWR CDC Surveill Summ. 1993: 1-19.

13. Silber E, Sonnenberg P, Koornhof HJ, Morris L, Saffer D. Dual infective pathology in patients with cryptococcal meningitis. Neurology 1998; 51: 1213-1215.

14. Mason PR, Jacobs MR, Fripp PJ. Serological survey of toxoplasmosis in the Transvaal. S Afr Med J 1974; 48: 1707-1709.

15. Schneider E, Schutte CHJ, BommerW. The prevalence of Toxoplasma gondii infection in women of different ethnic groups in Natal, South Africa. S Afr J Epidemiol Inf 1992; 7: 4145.

16. Sonnenberg $P$, Silber $E$, Jentsch $U$. Toxoplasmosis and HIV infection in Southern Africa. S Afr J Epidemiol Inf 1998; 13: 104-106.

17. Thornton CA, Houston S, Latif AS. Neurocysticercosis and human immunodeficiency virus infection - A possible association. Arch Neurol 1992; 49: 963-965.

Reprinted from QJM 2004; 97: 413-421, with permission. 